\title{
SHARP UPPER BOUNDS ON THE SIGNLESS LAPLACIAN SPECTRAL RADIUS OF STRONGLY CONNECTED DIGRAPHS ${ }^{1}$
}

\author{
Weige Xi AND LigONG WANG ${ }^{2}$ \\ Department of Applied Mathematics \\ School of Science, Northwestern Polytechnical University \\ Xi'an, Shaanxi 710072, P.R. China \\ e-mail: xiyanxwg@163.com \\ lgwangmath@163.com
}

\begin{abstract}
Let $G=(V(G), E(G))$ be a simple strongly connected digraph and $q(G)$ be the signless Laplacian spectral radius of $G$. For any vertex $v_{i} \in V(G)$, let $d_{i}^{+}$denote the outdegree of $v_{i}, m_{i}^{+}$denote the average 2-outdegree of $v_{i}$, and $N_{i}^{+}$denote the set of out-neighbors of $v_{i}$. In this paper, we prove that:

(1) $q(G)=d_{1}^{+}+d_{2}^{+},\left(d_{1}^{+} \neq d_{2}^{+}\right)$if and only if $G$ is a star digraph $\overleftrightarrow{K}_{1, n-1}$, where $d_{1}^{+}, d_{2}^{+}$are the maximum and the second maximum outdegree, respectively $\left({\overleftrightarrow{K_{1, n-1}}}\right.$ is the digraph on $n$ vertices obtained from a star graph $K_{1, n-1}$ by replacing each edge with a pair of oppositely directed arcs).

(2) $q(G) \leq \max \left\{\frac{1}{2}\left(d_{i}^{+}+\sqrt{d_{i}^{+^{2}}+8 d_{i}^{+} m_{i}^{+}}\right): v_{i} \in V(G)\right\}$ with equality if and only if $G$ is a regular digraph.

(3) $q(G) \leq \max \left\{\frac{1}{2}\left(d_{i}^{+}+\sqrt{d_{i}^{+2}+\frac{4}{d_{i}^{+}} \sum_{v_{j} \in N_{i}^{+}} d_{j}^{+}\left(d_{j}^{+}+m_{j}^{+}\right)}\right): v_{i} \in V(G)\right\}$. Moreover, the equality holds if and only if $G$ is a regular digraph or a bipartite semiregular digraph.

(4) $q(G) \leq \max \left\{\frac{1}{2}\left(d_{i}^{+}+2 d_{j}^{+}-1+\sqrt{\left(d_{i}^{+}-2 d_{j}^{+}+1\right)^{2}+4 d_{i}^{+}}\right):\left(v_{j}, v_{i}\right)\right.$ $\in E(G)\}$. If the equality holds, then $G$ is a regular digraph or $G \in \Omega$, where $\Omega$ is a class of digraphs defined in this paper.
\end{abstract}

Keywords: digraph, signless Laplacian spectral radius.

2010 Mathematics Subject Classification: 05C50, 15A18.

\footnotetext{
${ }^{1}$ The research was supported by the National Natural Science Foundation of China (Grant No. 11171273).

${ }^{2}$ Corresponding author.
} 


\section{REFERENCES}

[1] S..B. Bozkurt and D. Bozkurt, On the signless Laplacian spectral radius of digraphs, Ars Combin. 108 (2013) 193-200.

[2] B.K. Bulter and P.H. Siegel, Sharp bounds on the spectral radius of nonnegative matrices and digraphs, Linear Algebra Appl. 439 (2013) 1468-1478. doi:10.1016/j.laa.2013.04.029

[3] Y.Q. Chen and L.G. Wang, Sharp bounds for the largest eigenvalue of the signless Laplacian of a graph, Linear Algebra Appl. 433 (2010) 908-913. doi:10.1016/j.laa.2010.04.026

[4] S.Y. Cui, C.X. Tian and J.J. Guo, A sharp upper bound on the signless Laplacian spectral radius of graphs, Linear Algebra Appl. 439 (2013) 2442-2447. doi:10.1016/j.laa.2013.06.015

[5] K.Ch. Das, A characterization on graphs which achieve the upper bound for the largest Laplacian eigenvalue of graphs, Linear Algebra Appl. 376 (2004) 173-186. doi:10.1016/j.laa.2003.06.009

[6] K.Ch. Das, The Laplacian spectrum of a graph, Comput. Math. Appl. 48 (2004) 715-724.

doi:10.1016/j.camwa.2004.05.005

[7] K.Ch. Das, Maximizing the sum of the squares of the degrees of a graph, Discrete Math. 285 (2004) 57-66.

doi:10.1016/j.disc.2004.04.007

[8] E. Gudiño and J. Rada, A lower bound for the spectral radius of a digraph, Linear Algebra Appl. 433 (2010) 233-240.

doi:10.1016/j.laa.2010.02.012

[9] A.D. Güngör, K.C. Das and A.S. Çevik, Sharp upper bounds on the spectral radius of the signless Laplacian matrix of a graph, Appl. Math. Comput. 219 (2013) 5025-5032. doi:10.1016/j.amc.2012.11.039

[10] W.X. Hong and L.H. You, Spectral radius and signless Laplacian spectral radius of strongly connected digraphs, Linear Algebra Appl. 457 (2014) 93-113. doi:10.1016/j.laa.2014.05.007

[11] R.A. Horn and C.R. Johnson, Matrix Analysis (Cambridge University Press, New York, 1985). doi:10.1017/CBO9780511810817

[12] W.W. Lang and L.G. Wang, Sharp bounds for the signless Laplacian spectral radius of digraphs, Appl. Math. Comput. 238 (2014) 43-49. doi:10.1016/j.amc.2014.04.001

[13] M.H. Liu, B.L. Liu and B. Cheng, Ordering (signless) Laplacian spectral radii with maximum degrees of graphs, Discrete Math. 338 (2015) 159-163. doi:10.1016/j.disc.2014.09.007 
[14] C.S. Oliveira, L.S. de Lima, N.M.M. de Abreu and P. Hansen, Bounds on the index of the signless Laplacian of a graph, Discrete Appl. Math. 158 (2010) 355-360. doi:10.1016/j.dam.2009.06.023

[15] Y.L. Pan, Sharp upper bounds for the Laplacian graph eigenvalues, Linear Algebra Appl. 355 (2002) 287-295. doi:10.1016/S0024-3795(02)00353-1

[16] G.H. Xu, K.F. Fang and J. Shen, Bounds on the spectral radii of digraphs in terms of walks, Appl. Math. Comput. 219 (2012) 3721-3728. doi:10.1016/j.amc.2012.09.073

[17] G.L. Yu, Y.R. Wu and J.L. Shu, Sharp bounds on the signless Laplacian spectral radii of graphs, Linear Algebra Appl. 434 (2011) 683-687. doi:10.1016/j.laa.2010.09.029

Received 26 March 2015

Revised 13 January 2016

Accepted 13 January 2016 